\title{
BMJ open Exposure to bisphosphonates and risk of cancer: a protocol for nested case-control studies using the QResearch primary care database
}

To cite: Vinogradova $Y$, Coupland C, Hippisley-Cox J. Exposure to

bisphosphonates and risk of cancer: a protocol for nested case-control studies using the QResearch primary care database. BMJ Open 2012;2: e000548. doi:10.1136/ bmjopen-2011-000548

- Prepublication history for this paper is available online. To view these files please visit the journal online (http:// bmjopen.bmj.com).

Received 27 October 2011 Accepted 25 November 2011

This final article is available for use under the terms of the Creative Commons Attribution Non-Commercial 2.0 Licence; see http://bmjopen.bmj.com

Division of Primary Care, University of Nottingham, School of Community Health Sciences, Division of Primary Care, University Park, Nottingham, UK

Correspondence to Yana Vinogradova; yana. vinogradova@nottingham.ac. uk

\section{ABSTRACT}

Introduction: Bisphosphonates are becoming a common treatment for osteoporosis particularly after discovery of the association between hormone replacement therapy and increased risk of breast cancer. As osteoporosis develops with age, treatment is a long-term intervention. Randomised control trials typically have limited follow-up times, which restricts investigation of the effects of the drugs on risk of primary cancers. A few observational studies have demonstrated a reduced risk of breast cancer and possibly of endometrial cancer in bisphosphonate users. Two epidemiological studies have studied the effect of the drugs on oesophageal cancer but did not reach any definite conclusions. So far, no effects on colorectal and stomach cancer have been shown. This study will investigate the association of bisphosphonates with risks of the 10 most common primary cancers.

Methods and analysis: A series of nested case-control studies will be based on the general population using records from 660 UK general practices within the QResearch Database. Cases will be patients with primary cancers diagnosed between 1996 and 2011. Each case will be matched by age, sex, practice and calendar year to five controls, who are alive and registered with the practice at the time of diagnosis of the case. Exposure to bisphosphonates will be defined as at least one prescription during the study period. For the most common cancers with substantial numbers of observations, the effect of the duration of the treatment and different types of bisphosphonates will be studied. Conditional logistic regression will be applied to produce ORs adjusted for smoking status, socioeconomic status, ethnicity, cancer-specific co-morbidities and use of other medications

\section{INTRODUCTION}

Osteoporosis among the older people is a major problem leading to increased mortality and morbidity and high costs for health services. Thirty-five per cent of the European population aged 50 years and over

\section{ARTICLE SUMMARY}

Article focus

- Bisphosphonate use.

- Effect on incidence of cancer.

- Designing a study.

Key messages

- Series of case-control studies will examine possible associations between use of bisphosphonates and risk of cancer.

- Effect of dose, duration and different types of drug will be investigated.

- Results will be adjusted for a number of confounders.

Strengths and limitations of this study

- Large sample size.

- Based on the general populations.

- Based on routinely collected data.

- Prescriptions not actual use.

suffer from fractures caused by osteoporosis. ${ }^{1}$ Between 1980 and 1990, the use of hormone replacement therapy (HRT) was considered a preventive measure for postmenopausal osteoporotic fractures in women but, after a Women's Health Initiative trial report about increased risk of breast cancer, use of HRT fell significantly. ${ }^{2}$

As a treatment for postmenopausal osteoporosis, bisphosphonates were introduced in the 1990s, and prescribing of them has increased substantially and continually. HRT (raloxifene) and the use of calcitonin and strontium ranelate ${ }^{3}$ are still considered to be options for the treatment of osteoporosis, but according to the UK National Institute for Health and Clinical Excellence guidelines, ${ }^{45}$ recommending bisphosphonates as a firstline therapy for osteoporosis bisphosphonates have become the most commonly prescribed drug.

The proportion of the female population in the UK eligible for treatment varies 
between $24 \%$ and $47 \%$, depending on age. ${ }^{6}$ The drugs increase bone mass and reduce the risk of fracture, but these effects become significant only after $6-36$ months of use depending on the type of drug. ${ }^{7}$ Bisphosphonates bind to bone and, depending on type, can be released for up to ten more years after treatment ceases. ${ }^{8}$

The first use of bisphosphonates in the 1970s was in oncology. They were used for the treatment and prevention of skeletal disorders associated with multiple myeloma and bone metastases from breast, prostate, lung and kidney cancers and other solid tumours. Bisphosphonates have also been used for glucocorticoidinduced osteoporosis. ${ }^{7}$

There is preclinical evidence for the anti-tumour effects of bisphosphonates because of their anti-resorptive properties. ${ }^{9}$ Bone is a good environment for tumour cells because of a number of growth factors. Osteoclasts affect release of soluble growth factors and so promote tumour cells. Bisphosphonates accumulated in bones inhibit osteoclast-mediating bone resorption with significant clinical effect. The drugs also demonstrate anti-tumour effects in vitro by inhibiting angiogenesis (adhesion, invasion and proliferation) and inducing apoptosis. The cancers studied in vitro were breast, prostate, myeloma, pancreatic and osteosarcoma. ${ }^{10}$ These preclinical studies, however, were conducted with concentrations far higher than those used for treating patients with bone metastates. ${ }^{11}$

Although the anti-tumour properties of bisphosphonates are being considered for prevention of bone metastases and a few clinical trials have demonstrated the efficacy of bisphosphonates in women with earlystage breast cancer, ${ }^{12}$ they have been little studied in relation to the development of other primary cancers. Four epidemiological studies concentrating on breast cancer have shown positive effects for bisphosphonates: $32 \%$ RR reduction in postmenopausal women (HR 0.68, $95 \%$ CI 0.52 to 0.88$),{ }^{13} 33 \%$ decreased risk in current users, women aged 20-69 years (OR $0.67,95 \%$ CI 0.51 to 0.89$),{ }^{14} 39 \%$ risk reduction in patients taking bisphosphonates for at least 1 year (OR 0.61, 95\% CI 0.50 to 0.76$)^{15}$ and $47 \%$ risk reduction after start of alendronate (HR $0.53,95 \%$ CI 0.38 to 0.73 ) and $20 \%$ for etidronate (HR $0.80,95 \%$ CI 0.73 to 0.89 ). ${ }^{16}$ A study looking at the risk of endometrial cancer has also shown a $30 \%$ decrease associated with bisphosphonate use, but it was not statistically significant (OR 0.7, 95\% CI 0.4 to 1.2$){ }^{17}$

Because bisphosphonates are associated with shortterm gastrointestinal adverse effects, ${ }^{8}$ an adverse effect on risk of oesophageal cancer might be expected. The first publication about the association was from the US Food and Drug Administration Adverse Event Reporting System, which listed 23 cases of oesophageal cancer in users of oral alendronate between 1995 and 2008. ${ }^{18} \mathrm{~A}$ further observational study, based on 13678 bisphosphonate users matched to 27365 non-users, identified 37 oesophageal cancers and 48 gastric cancers and showed reduced risks for oesophageal and gastric cancers (HR $0.35,95 \%$ CI 0.14 to 0.85 and HR $1.23,95 \%$ CI 0.68 to 2.22 , respectively). ${ }^{19}$

A case-control study looking at 2954 cases of oesophageal, 2018 cases of gastric and 10641 cases of colorectal cancers, based on the General Practice Research Database, demonstrated a $30 \%$ increased risk of oesophageal cancer in patients with at least one prescription for bisphosphonates (OR 1.30, 95\% CI 1.02 to 1.66$)^{20}$ but did not find a significant effect on risk of gastric or colorectal cancers (OR 0.87, 95\% CI 0.64 to 1.19 and OR $0.87,95 \%$ CI 0.77 to 1.00 , respectively). A cohort study based on the General Practice Research Database did not find any significant association between bisphosphonate use and risk of gastric or oesophageal cancers ${ }^{21}$ (combined HR $0.96,95 \%$ CI 0.74 to 1.25 , for oesophageal cancer only HR $1.07,95 \%$ CI 0.77 to 1.49 ). As for colorectal cancer, an Israeli study showed a significantly decreased risk in patients taking bisphosphonates for more than a year (RR $0.50,95 \%$ CI 0.25 to 0.67$).{ }^{22} \mathrm{~A}$ Danish study looked at gastrointestinal cancers and reported an excess risk of oesophageal cancer associated with use of alendronate (HR 2.10, 95\% CI 1.01 to 4.35 ) and etidronate (HR 1.99, 95\% CI 1.24 to 3.18 ) and a possible protective effect of higher doses for colorectal cancer (HR $0.29,95 \%$ CI 0.14 to 0.62 ). ${ }^{23}$ So far no epidemiological studies have investigated associations with risks of other common cancers for bisphosphonate users. A few randomised controlled trials - the longest for up to 10 years $^{24-26}$ - have studied the effect of the drugs on skeletal properties and general adverse effects, but none of them have considered cancer as a consequence of osteoporotic therapy. A cohort study in patients treated for osteoporosis including bisphosphonates is currently enrolling participants to explore a number of adverse events in the next 5 years. ${ }^{27}$ This is the only study where malignancies form part of the secondary outcome measures.

Our aim is to examine possible associations between use of bisphosphonates and risk of a range of common cancers in a large community sample, including the effect of dose, duration and type of drug.

\section{METHODS AND ANALYSIS}

\section{Sample selection}

This will be a study using the QResearch primary care research database, which consists of routinely collected data from general practitioner clinical computer systems. The contributing practices, which comprise around $7 \%$ of all UK general practices, use the Egton Medical Information System. QResearch is one of the largest general practice databases, containing anonymised clinical records for over 13 million patients registered with 660 UK general practices. The information recorded on the database includes patient demographics (year of birth, sex, socio-demographic data derived from UK census 2001), characteristics (height, weight, smoking status), clinical diagnoses, symptoms 
and prescribed medications (including repeat prescriptions). Detailed analyses, including age and sex distribution, birth rates and death rates, have been undertaken and have shown good correspondence with other sources ${ }^{28}$ and demonstrated the accuracy and completeness of the data. ${ }^{29}$

An open cohort of patients will be identified, 30 years or older, registered with the study practices during the study period, between 1 January 1996 and 1 July 2011. Temporary residents will be excluded. Cases will be incident cases of cancer identified during the study period, and these will include the 10 most common cancers. Cases with any previous cancer diagnosis will be excluded. Cases with secondary cancers (READ codes: B56, B57, B58) will be excluded. The right censor date will be the earliest of the following: date of diagnosis of cancer, date of death, date of leaving the practice, date of the latest download of data, the study end date.

\section{Cases and controls}

Each case will be matched to five controls, who are alive and registered with the practice at the time of diagnosis of the case. Controls will be matched on age, sex, practice and calendar year using incidence density sampling. Controls will be allocated an index date, which is the date on which their matched case was first diagnosed with cancer. Controls with a diagnosis of any cancer before the index date will be excluded.

Cases and controls with a record of mastectomy before their first prescription of bisphosphonates will be excluded since this treatment is likely to indicate a previous diagnosis of breast cancer with further bone metastases. For breast cancer, only female patients will be included. All patients with Paget's disease will be excluded as the treatment for this condition is administered in higher doses and for much longer periods (typically 2 weeks for osteoporosis against 6 months for Paget's). Patients with prescriptions for the bisphosphonates licensed not for osteoporosis but for malignancies (zoledronic acid, clodronate and daily use of ibandronate) will also be excluded.

For the main analysis, cases and controls will be included if they have complete records for at least 2 years before the index date. A subset of cases and controls with at least 6 years of records will be used for further analyses.

The risks of any cancer and of the 10 most common cancers will be determined for patients prescribed bisphosphonates and compared with the risks for patients not prescribed these drugs. The 'most common' cancers have been selected because they have this status in the UK. ${ }^{30}$ They are breast cancer (women, B34), prostate cancer (men, B46), lung cancer (B22), colorectal cancer (B13, B14), haematological malignancies (B6), bladder cancer (B49), melanoma (B32), gastric cancer (B11), pancreatic cancer (B17) and oesophageal cancer (B10). As osteoporosis might be an early symptom of possible myeloma, it will be analysed separately from lymphoma and leukaemia. The commoner female cancers (ovary (B44), uterus (B43) and cervix (B41)) will also be considered.

\section{Interventions}

Exposure to drugs for osteoporosis will be determined based on all prescriptions for bisphosphonates and other drugs before the index date (date of diagnosis or equivalent date for controls) within the observation period (from the date of entry into QResearch to the index date). The bisphosphonates to be included are identified in the British National Formulary section 6.6.2 as treatment for osteoporosis ${ }^{3}$ : alendronate $(5-10 \mathrm{mg}$ daily or $70 \mathrm{mg}$ weekly), etidronate ( $400 \mathrm{mg}$ daily for 14 days in 90-day cycles), ibandronate $(150 \mathrm{mg}$ a month or intravenous $3 \mathrm{mg} / 3$ months) and risedronate ( $5 \mathrm{mg}$ daily).

The cumulative exposure to bisphosphonates will be assessed by extracting duration of the prescribed days' supply and summarising it for each patient. For drugs prescribed in cycles, the length of a cycle will be considered as duration of a prescription, for example, etidronate prescription for 2 weeks will be assessed as a 90-day prescription duration. The same approach will be applied to intravenous infusion, considering the recommended interval between injections as the duration of a prescription (eg, 3 months for ibandronate). The cumulative exposure to bisphosphonates will be estimated by extracting the duration for every prescription, and for groups of prescriptions with interprescription gaps of $<60$ days, overall course times will be calculated from the start of the first prescription to the end of the last prescription.

As bisphosphonates can be released for months after a treatment, total exposure to bisphosphonates will be estimated as the time between the first prescription and the end time for the last prescription.

Because bisphosphonates and other osteoporosis treatment drugs are prescribed for years, long-term users and short-term users will be distinguished, as treatment of the latter might have been for accidental or clinical fractures or for better integration of biomaterial or implants. The effect of bisphosphonates on treating fractures varies from 6 to 36 months, for example 12 months for risedronate and 24 months for alendronate. ${ }^{7}$

There are three regimens for bisphosphonate use: daily, once weekly and once monthly. Daily use has been shown to have lower adherence than weekly use. ${ }^{31}$ Another reason for investigating regimens is that, particularly for gastrointestinal organs, there might be a marked difference between the effects of daily and weekly exposure to bisphosphonates, with associated effects on risks for oesophageal, gastric and colorectal cancers.

Bisphosphonate use will be categorised in a number of ways. The main analyses will compare patients having no prescriptions for the drugs with patients with at least one prescription for any bisphosphonate. The effect of prescribing for short-term ( $<12$ months) and long-term (at least 12 months) periods will then be analysed, as well as the effect of regimen: daily or weekly/monthly. 
If there are a sufficient number of observations, further analyses will be run for the cumulative exposure (cumulative duration of all prescriptions) and the exposure time to bisphosphonate (the time period between the first prescription and the end time for the last prescription). The subset of data with at least 6 years of records will be analysed using following categorisations: no use, $<180$ days, 180 days up to 12 months, 12-24 months and $\geq 25$ months. A test for trend will be performed using the actual number of months.

Timing will be categorised as: no use before diagnosis, used within 1-2 years before the index date and used $>2$ years before the index date. The interaction of timing and terms of treatment will also be examined, categorised as: no use before diagnosis; used within 1-2 years before the index date, short-term use; used within 1-2 years before the index date, long-term use; used $>2$ years before the index date, short-term use and used $>2$ years before the index date, long-term use.

If there are any variations in dose of bisphosphonates, it will be categorised as low ( $<67 \%$ of dose recommended by dose) and normal/high ( $>66 \%$ of recommended dose).

The two main types of bisphosphonates-simple bisphosphonates (etidronate) and nitrogen containing $^{7}$-will be analysed as there are two different mechanisms of action for the drugs. If there are sufficient numbers, the data will also be analysed by individual drug.

Because prescriptions in the year before the index date might be associated with an early symptom of cancer before a recorded diagnosis, sensitivity analyses ignoring all prescriptions in the last year before the index date will be run. The results from these analyses will highlight any attenuation of the protective effects of bisphosphonates or any increases in magnitudes of harmful effects. A sensitivity analysis on the main analysis will also be run, defining the use of bisphosphonates as at least two prescriptions within the observation period. The analyses will be repeated on a subgroup of patients with at least 6 years of records to estimate the long-term effect of bisphosphonate use.

The other drugs for osteoporosis to be included are strontium ranelate, raloxifene and calcitonin. As there will not be enough observations to analyse each drug individually, they will be combined and included in the analyses as other treatment for osteoporosis. A patient will be considered as a user if they have at least one prescription of any of those drugs in their records before the index date.

\section{Confounding factors}

All the analyses will include potential confounders which are established as risk factors for cancer: body mass index ${ }^{32}$ (continuous variable, at the date closest to 1 year before the diagnosis and recorded before the index date); smoking status ${ }^{33}$ (current smoker-light (1-9 cigarettes/day), medium (10-19 cigarettes/day) and heavy ( $\geq 20$ cigarettes/day); ex-smoker and non-smoker); excessive alcohol consumption ${ }^{34}$ using Read codes for alcohol status (only if it is a significant confounder for the sample); socioeconomic status ${ }^{35}$ (Townsend score in fifths) and ethnicity ${ }^{36}$ (Caucasian, African-American, Asian and other). The analysis will also adjust for osteoporosis history, ${ }^{37}$ including diagnosis of osteoporosis or osteopenia or previous fractures, use of drugs increasing risk of fracture (systemic corticosteroids and proton pump inhibitors ${ }^{38}$ ), use of anti-inflammatory drugs ${ }^{39}$ (traditional non-steroidal, cyclooxygenase 2 inhibitors and aspirin) ${ }^{40}$ and use of vitamin D. ${ }^{41}$

Co-morbidities which affect risks of cancer will also be included: rheumatoid arthritis ${ }^{42}$ for any cancer; hypertension $^{43}$ for uterine cancer and diabetes and glucose intolerance for pancreatic, ${ }^{44}$ uterine $^{45}$ and colorectal ${ }^{46}$ cancers. Analyses of colorectal, oesophageal, gastric and pancreatic cancers will be adjusted for gastrointestinal disorders $^{47}$ if diagnosed before the first use of bisphosphonates or 12 months before the index date, whichever is earlier: upper gastrointestinal disease (dysphagia, oesophagitis, gastrooesophageal reflux disease, hiatus hernia, oesophageal ulcers, Barrett's oesophagus, gastritis, duodenitis, peptic ulcers, dyspepsia); Crohn's disease, ulcerative colitis, and pancreatitis. Bladder cancer analyses will include renal impairment $^{48}$ (diagnostic code for chronic kidney disease) if diagnosed before the first use of bisphosphonates or 12 months before the index date, whichever is earlier. Breast cancer analyses will also include previous benign breast disease (fibrocystic disease, intraductal papilloma or fibroadenoma). ${ }^{49}$ The results will also be adjusted for family history of cancer $^{50}$ (this will vary according to the cancer under consideration) if recorded 6 months before the index date. This is to reduce family recall bias as cases are more likely to report a family history of cancer around the time of diagnosis. ${ }^{51}$

Because use of some drugs might be associated with increased risk of some cancers, use of $\mathrm{HRT}^{52}$ and oral contraceptives $^{53}$ for breast, uterine, ovarian and cervical cancers will also be included. Use of acid suppression drugs $^{54}$ (including $\mathrm{H} 2$ antagonists (BNF 1.3.1), proton pump inhibitors (BNF 1.3.5) and antacids (BNF 1.1.1)) will be added for gastrointestinal cancer analyses. If there are enough observations, use of those drugs will be categorised by the number of prescriptions within the observation period: none, fewer than 12 prescriptions, 12-24 prescriptions, 25-48 prescriptions and $>49$ prescriptions.

\section{Statistical analysis}

Conditional logistic regression will be used to estimate OR with $95 \%$ CIs for cancer of any site and each of the 10 most common cancers and three additional female cancers and their matched controls. The initial analysis model will determine the unadjusted ORs for each cancer associated with bisphosphonate prescriptions. A multivariable model will determine the OR for each 
cancer associated with bisphosphonate prescriptions, adjusted for the potential confounding effects of the variables listed above.

As body mass index, smoking status and alcohol consumption may be important confounders but have non-negligible numbers of missing data, multiple imputation will be used to impute the missing values. Ten imputed data sets will be created. Index year, case/ control status, years of records, potential confounders and exposure to bisphosphonates and other drugs will be included. For comparison, analyses with missing data treated as separate categories will also be carried out.

Stata V.11 will be used for all the analyses. A $1 \%$ significance level will be used to account for the multiple outcomes.

\section{Sample size calculation}

As different types of cancer may have different risks associated with bisphosphonate use, analyses will require number of cases to relate to each type of cancer. All available data from QResearch will be used. Our calculations are based on the exposure to bisphosphonates in the proposed data extraction for $6.8 \%$ of women and $1.8 \%$ of men. For non-gender-specific cancers, the total proportion of users is estimated as $4.2 \%$. To detect an OR of 0.87 (for colorectal or stomach cancers ${ }^{20}$ ), 22322 cases will be needed. To detect an OR of 1.3 (for oesophageal cancer $^{20}$ ), 5208 cases will be needed. To detect an OR of 0.70 (for breast ${ }^{14}$ or uterus ${ }^{17}$ cancers), 2382 female cases will be needed. For prostate cancer, to detect 30\% increase (or decrease) in risk, 11773 (or 8686) male cases will be needed. For other cancers, a detection of $30 \%$ risk decrease will require 3785 cases. All calculations are done for matched sets of cases and controls, with 4.5 matched controls per case, an estimated coefficient for exposure between matched cases and controls of 0.2 , a power of $80 \%$ and a significance level of $1 \%$.

Acknowledgements We acknowledge the contribution of Egton Medical Information System and the University of Nottingham for expertise in creating and maintaining QResearch and to the Egton Medical Information System practices, which contribute data without whom this research would not be possible.

Funding This work has been funded by the Division of Primary Care of University of Nottingham. Apart from that, this research received no specific grant from any funding agency in the public, commercial or not-for-profit sectors.

Competing interests All authors have completed the Unified Competing Interest form at www.icmje.org/coi_disclosure.pdf (available on request from the corresponding author) and declare no support from any additional organisation for the submitted work. $\mathrm{JH}-\mathrm{C}$ is professor of clinical epidemiology at the University of Nottingham and unpaid director of QResearch, a not-for-profit organisation, which is a joint partnership between the University of Nottingham and Egton Medical Information System (commercial IT supplier for $60 \%$ of general practices in the UK). JH-C is also a paid director of ClinRisk Limited, which produces open and closed source software to ensure the reliable and updatable implementation of clinical risk algorithms within clinical computer systems to help improve patient care. CC is associate professor of medical statistics at the University of Nottingham and a paid consultant statistician for ClinRisk Limited; no other relationships or activities that could appear to have influenced the submitted work.
Ethics approval This protocol has been independently peer-reviewed by the QResearch Scientific Board and has been reported to Trent Research Ethics Committee in accordance with the agreed procedure. A full report containing the study findings will be prepared and a paper based on the report will be submitted to a peer-reviewed journal.

Contributors $\mathrm{JH}-\mathrm{C}$ had the original idea for this study. CC contributed to the development of the idea. YV reviewed the literature, contributed to the study design and wrote the draft of the manuscript. JH-C and CC critically reviewed the paper. YV is the guarantor of the study. All authors approved the submitted version.

Provenance and peer review Not commissioned; externally peer reviewed.

Data sharing statement It will be possible to access the data after the publication of the results but only on premises of the University of Nottingham. The full protocol and statistical code will be available from the authors after the publication of the results.

\section{REFERENCES}

1. Johnell O, Kanis JA. An estimate of the worldwide prevalence and disability associated with osteoporotic fractures. Osteoporos Int 2006;17:1726-33.

2. Watson J, Wise L, Green J. Prescribing of hormone therapy for menopause, tibolone, and bisphosphonates in women in the UK between 1991 and 2005. Eur J Clin Pharmacol 2007;63:843-9.

3. Joint Formulary Committee. British National Formulary London. British Medical Association and Royal Pharmaceutical Society of Great Britain. 62 ed. London: BMJ Group and Pharmaceutical Press 2011.

4. National Institute for Health and Clinical Excellence. Alendronate, etidronate, risedronate, strontium ranelate and raloxifen for preventing bone fractures in postmenopausal women with osteoporosis who have not had a fracture. information about NICE technology appraisal guidance. London: National Institute for Health and Clinical Excellence, 2008;160.

5. National Institute for Health and Clinical Excellence. Alendronate, etidronate, risedronate, strontium ranelate and teriparatide for preventing bone fractures in postmenopausal women with osteoporosis who have already had a fracture. Information about NICE technology appraisal guidance. 2008;161.

6. Kanis J, McCloskey E, Johansson H, et al. Case finding for the management of osteoporosis with FRAX ${ }^{\circledR}$-assessment and intervention thresholds for the UK. Osteoporos Int 2008;19:1395-408.

7. Russell RG, Watts NB, Ebetino FH, et al. Mechanisms of action of bisphosphonates: similarities and differences and their potential influence on clinical efficacy. Osteoporos Int 2008;19:733-59.

8. Watts NB, Diab DL. Long-Term use of bisphosphonates in osteoporosis. J Clin Endocrinol Metab 2010;95:1555-65.

9. Guise TA. Antitumor effects of bisphosphonates: promising preclinical evidence. Cancer Treat Rev 2008;34(Suppl 1):S19-24.

10. Croucher $P$, Jagdev S, Coleman R. The anti-tumor potential of zoledronic acid. Breast 2003;12(Suppl 2):S30-6.

11. Santini D, Fratto ME, Galluzzo S, et al. Are bisphosphonates the suitable anticancer drugs for the elderly? Crit Rev Oncol Hematol 2009;69:83-94.

12. Gnant M. Can oral bisphosphonates really reduce the risk of breast cancer in Healthy women? J Clin Oncol 2010;28:3548-51.

13. Chlebowski RT, Chen Z, Cauley JA, et al. Oral bisphosphonate use and breast cancer incidence in postmenopausal women. J Clin Oncol 2010;28:3582-90.

14. Newcomb PA, Trentham-Dietz A, Hampton JM. Bisphosphonates for osteoporosis treatment are associated with reduced breast cancer risk. Br J Cancer 2010;102:799-802.

15. Rennert G, Pinchev M, Rennert HS. Use of bisphosphonates and risk of postmenopausal breast cancer. J Clin Oncol 2010;28:3577-81.

16. Vestergaard $\mathrm{P}$, Fischer L, Mele $\mathrm{M}$, et al. Use of bisphosphonates and risk of breast cancer. Calcif Tissue Int 2011;88:255-62.

17. Fortuny J, Sima C, Bayuga S, et al. Risk of endometrial cancer in relation to medical conditions and medication use. Cancer Epidemiol Biomarkers Prev 2009;18:1448-56.

18. Wysowski DK. Reports of esophageal cancer with oral bisphosphonate use. N Engl J Med 2009;360:89-90.

19. Abrahamsen B, Eiken P, Eastell R. More on reports of esophageal cancer with oral bisphosphonate use [letter]. $N$ Engl J Med 2009;360:1789.

20. Green J, Czanner G, Reeves G, et al. Oral bisphosphonates and risk of cancer of oesophagus, stomach, and colorectum: case-control analysis within a UK primary care cohort. BMJ 2010;341:c4444. 
21. Cardwell CR, Abnet CC, Cantwell MM, et al. Exposure to oral bisphosphonates and risk of esophageal cancer. JAMA 2010;304:657-63.

22. Rennert G, Pinchev M, Rennert HS, et al. Use of bisphosphonates and reduced risk of colorectal cancer. J Clin Oncol 2011;29:1146-50.

23. Vestergaard P. Occurrence of gastrointestinal cancer in users of bisphosphonates and other antiresorptive drugs against osteoporosis. Calcif Tissue Int 2011;89:434-41.

24. Mellström DD, Sörensen $\mathrm{OH}$, Goemaere $\mathrm{S}$, et al. Seven years of treatment with risedronate in women with postmenopausal osteoporosis. Calcif Tissue Int 2004;75:462-8.

25. Black DM, Schwartz AV, Ensrud KE, et al. Effects of continuing or stopping alendronate after 5 Years of treatment: the Fracture Intervention Trial Long-term Extension (FLEX): a randomized trial. JAMA 2006;296:2927-38.

26. Bone HG, Hosking D, Devogelaer JP, et al. Ten years' experience with alendronate for osteoporosis in postmenopausal women. $\mathrm{N} \mathrm{Engl}$ J Med 2004:350:1189-99.

27. Pfizer. Bazedoxifene Post Approval Safety Study (PASS) in the European Union (EU). 2011. B1781044. http://www.clinicaltrials.gov

28. Hammersley V, Hippisley-Cox J, Wilson A, et al. A comparison of research general practices and their patients with other practices-cross sectional survey in Trent. Br J Gen Pract 2002;52:463-8.

29. Hippisley-Cox J, Hammersley V, Pringle M, et al. How useful are General Practice databases for research? Analysis of their accuracy and completeness in one research network. Health Inform J 2004;10:91-109.

30. Westlake S. Cancer incidence and mortality in the United Kingdom and constituent countries, 2004-06. Health Stat Q. 2009 Autumn; 43:56-62.

31. Recker RR, Gallagher R, MacCosbe PE. Effect of dosing frequency on bisphosphonate medication adherence in a large longitudinal cohort of women. Mayo Clin Proc 2005;80:856-61.

32. Henderson KD, Bernstein L. Etiology of Cancer: Obesity and Physical Activity. DeVita, Hellman, and Rosenberg's Cancer: Principles \& Practice of Oncology. Wolters Kluwer/Lippincott Williams \& Wilkins, Philadelphia, 2008:239-44.

33. Hecht SS. Etiology of Cancer: Tobacco. DeVita, Hellman, and Rosenberg's Cancer: Principles \& Practice of Oncology. Wolters Kluwer/ Lippincott Williams \& Wilkins, Philadelphia, 2008:147-55.

34. Schütze $M$, Boeing $\mathrm{H}$, Pischon $\mathrm{T}$, et al. Alcohol attributable burden of incidence of cancer in eight European countries based on results from prospective cohort study. BMJ 2011;342:d1584.

35. Forman D. Cancer Incidence by Deprivation. England, 1995-2004. 2008. http://wwwncinorguk/publications/reports/defaultaspx, http:// www.ncin.org.uk/publications/reports/default.aspx

36. Ferlay J, Shin HR, Bray F, et al. Estimates of worldwide burden of cancer in 2008: GLOBOCAN 2008. Int J Cancer 2010;127:2893-917.
37. McGlynn KA, Gridley G, Mellemkjaer L, et al. Risks of cancer among a cohort of 23,935 men and women with osteoporosis. Int J Cancer 2008;122:1879-84.

38. Yang YX, Lewis JD, Epstein S, et al. Long-term proton pump inhibitor therapy and risk of Hip fracture. JAMA 2006;296:2947-53.

39. Coussens LM, Werb Z. Inflammation and cancer. Nature 2002;420:860-7.

40. González-Pérez A, Garcia Rodriguez L, Lopez-Ridaura R. Effects of non-steroidal anti-inflammatory drugs on cancer sites other than the colon and rectum: a meta-analysis. BMC Cancer 2003;3:28.

41. Mocellin S. Vitamin D and cancer: deciphering the truth. Biochim Biophys Acta 2011;1816:172-8.

42. Thomas E, Brewster DH, Black RJ, et al. Risk of malignancy among patients with rheumatic conditions. Int J Cancer 2000;88:497-502.

43. Bangalore S, Kumar S, Kjeldsen SE, et al. Antihypertensive drugs and risk of cancer: network meta-analyses and trial sequential analyses of 324,168 participants from randomised trials. Lancet Oncol 2011;12:65-82.

44. Vincent A, Herman J, Schulick R, et al. Pancreatic cancer. Lancet 2011;378:607-20.

45. Burbos N, Musonda P, Giarenis I, et al. Predicting the risk of endometrial cancer in postmenopausal women presenting with vaginal bleeding: the Norwich DEFAB risk assessment tool. $\mathrm{Br} J$ Cancer 2010;102:1201-6.

46. Stattin $\mathrm{P}, \mathrm{Björ} \mathrm{O}$, Ferrari $\mathrm{P}$, et al. Prospective study of hyperglycemia and cancer risk. Diabetes Care 2007;30:561-7.

47. Rustgi AK. Cancers of the Gastrointestinal Tract. DeVita, Hellman, and Rosenberg's cancer: Principles \& Practice of Oncology. Wolters Kluwer/Lippincott Williams \& Wilkins, Philadelphia, 2008:989-1313

48. Perazella MA, Markowitz GS. Bisphosphonate nephrotoxicity. Kidney Int 2008;74:1385-93.

49. Hartmann LC, Sellers TA, Frost MH, et al. Benign breast disease and the risk of breast cancer. N Engl J Med 2005;353:229-37.

50. Mai PL, Garceau AO, Graubard BI, et al. Confirmation of family cancer history reported in a population-based survey. J Natl Cancer Inst 2011;103:788-97.

51. Chang ET, Smedby KE, Hjalgrim $\mathrm{H}$, et al. Reliability of self-reported family history of cancer in a large case-control study of lymphoma. $J$ Natl Cancer Inst 2006;98:61-8.

52. Heiss $G$, Wallace R, Anderson GL, et al. Health risks and benefits 3 years after stopping randomized treatment with estrogen and progestin. JAMA 2008;299:1036-45.

53. Yager JD, Davidson NE. Estrogen carcinogenesis in breast cancer. $N$ Engl J Med 2006;354:270-82.

54. García Rodríguez LA, Lagergren J, Lindblad M. Gastric acid suppression and risk of oesophageal and gastric adenocarcinoma: a nested case control study in the UK. Gut 2006;55:1538-44. 